\title{
Experimental study of relativistic effects in the dp breakup reac- tion using the WASA detector
}

\author{
B. Kłos ${ }^{1, a}$, I. Ciepał ${ }^{2}$ B. Jamróz ${ }^{1}$, G. Khatri ${ }^{2}$, S. Kistryn ${ }^{2}$, A. Kozela ${ }^{3}{ }^{2}$ A. Magiera ${ }^{2}$, W. Parol $^{2}$, \\ I. Skwira-Chalot ${ }^{4}$, and E. Stephan ${ }^{1}$, \\ the WASA-at-COSY Collaboration \\ ${ }^{1}$ University of Silesia, PL-40007 Katowice, Poland \\ 2 Jagiellonian University, PL-30059 Kraków, Poland \\ ${ }^{3}$ Institute of Nuclear Physics PAN, PL-31342 Kraków, Poland \\ ${ }^{4}$ Faculty of Physics, University of Warsaw, Warsaw, Poland
}

\begin{abstract}
An experiment to investigate the ${ }^{1} \mathrm{H}(\vec{d}, p p) n$ breakup reaction at $340 \mathrm{MeV}$, $360 \mathrm{MeV}$ and $400 \mathrm{MeV}$ deuteron beam energy has been performed at the Cooler Synchrotron COSY-Jülich with the WASA detector. The main goal was to study of various aspects of few-nucleon dynamics in the medium energy region, with a particular emphasis on relativistic effects and their interplay with three nucelon forces. The almost $4 \pi$ geometry of the WASA detector gives an unique possibility to study the different aspects of nucleon-nucleon dynamics in the three nucleon system. The preliminary analysis of the collected data is presented.
\end{abstract}

\section{Introduction}

Few-nucleon systems are ideal laboratories to study nuclear forces. Among them, the system composed of three nucleons $(3 N)$ is the simplest non-trivial environment, in which various models of the nucleon-nucleon $(N N)$ interaction can be tested. The observables for the deuteron breakup in collision with protons can be calculated using modern realistic pairwise nucleon-nucleon $(N N)$ interactions, combined with a suitable model of $3 N$ forces [1]. Moreover, the two- and three-nucleon interactions can be modeled within the coupled-channel (CC) framework by an explicit treatment of the $\Delta$-isobar [2]. Alternatively, the dynamics is treated within the Chiral Perturbation Theory (ChPT), so far at the next-to-next-to-leading order with all relevant $N N$ and $3 N$ contributions taken into account [3]. The above listed calculations include different pieces of the nucleon-nucleon dynamics like the three nucleon force $3 \mathrm{NF}$, the long-range Coulomb interaction or relativistic effects, which reveal their influence at different parts of phase space. Especially, cross section observables are very sensitive to all these effects. Previous measurements of the cross sections at different deuteron beam energies have demonstrated that an inclusion of $3 N$ and Coulomb forces in the theoretical calculations improves the description of the experimental data [4].

In recent years the relativistic treatment of the breakup reaction in $3 N$ system was developed using the $N N$ potential [5] and this approach has also been extended for calculations including $3 \mathrm{NF}$ [6]. It

ae-mail: barbara.klos@us.edu.pl

This is an Open Access article distributed under the terms of the Creative Commons Attribution License 2.0, which permits unrestricted use, distribution, and reproduction in any medium, provided the original work is properly cited. 
was shown that in some particular regions of the breakup phase-space, relativistic effects can increase or decrease the calculated breakup cross sections by up to $60 \%$ and even more. At the same time the effects of $3 \mathrm{NF}$ may change certain observables by a similar factor. The relativistic effects and their interplay with $3 \mathrm{NF}$ become more important with increasing available energy in the three nucleon system. Therefore the investigations at relatively high energies are important to confirm the theoretical predictions for relativistic effects and to unambiguously fix a relevance of the $3 \mathrm{NF}$.

\section{Experiment}

The experiment using the ${ }^{1} \mathrm{H}(\vec{d}, p p) n$ breakup reaction at $340 \mathrm{MeV}, 360 \mathrm{MeV}$ and $400 \mathrm{MeV}$ deuteron beam energy has been performed in January 2013 at the Cooler Synchrotron COSY-Jülich with the WASA-at-COSY detector. The basic information of the experiment is shown in Table 1. The almost $4 \pi$ geometry of WASA (Wide Angle Shower Apparatus) gives an unique possibility to study various aspects in the three nucleon $(3 N)$ system, which appear in different phase-space regions.

The WASA detector consists of four main components: Central Detector (CD), Forward Detector (FD), Pellet Target Device and the Scattering Chamber (see Figure 1).

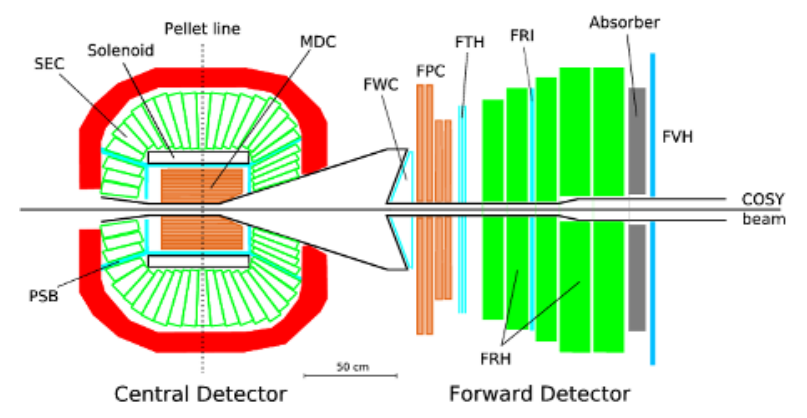

Figure 1. Schematic view of the detection system

The target components are situated on top of the platform of the CD. This target provides a narrow stream of frozen hydrogen or deuteron droplets pellets with diameters of about $20 \mu \mathrm{m}$.

The $\mathrm{CD}$ surrounds the interaction region and covers the region of the polar angles from $20^{\circ}$ to $169^{\circ}$. It consists of four elements: Mini Drift Chamber (MDC), Plastic Scintillator Barrel (PSB), Scintillator Electromagnetic Calorimeter (SEC) and Super Conduction Solenoid (SCS).

The FD covers the region of the polar angles from deuterons of $3^{\circ}$ to $18^{\circ}$. It consists of a set of plastic scintillators for the identification of charged hadrons and track reconstruction: Forward Window Counter (FWC), Forward Proportional Chamber (FPC), Forward Trigger Hodoscope (FTH), The Forward Range Hodoscop (FRH) and Forward Veto Hodoscope (FVH).

The basic informations of these dp breakup experiments are shown in Table 1.

\section{Data Analysis}

The first step of data analysis is the identification of interesting events, i.e. two protons from the breakup process and deuteron-proton pairs from elastic scattering channel. The particle identification is based on the $\Delta E-E$ technique. In the whole range of energies, a clear separation between loci of protons and deuterons is observed (Figure 2). 
INPC 2013

Table 1. General information of $d-p$ experiment

\begin{tabular}{|l|l|}
\hline deuteron beam energy & $340,380,400 \mathrm{MeV}$ \\
\hline reaction channels & $d p \rightarrow d p$ \\
& $d p \rightarrow p p n$ \\
& $d p \rightarrow{ }^{3} \mathrm{He}+\gamma$ \\
& $d p \rightarrow d p \gamma$ \\
\hline luminosity & $\sim 10^{29} / \mathrm{s} / \mathrm{cm}^{2}$ \\
\hline deuterons in a flat top & $(1.3-1.4) \cdot 10^{8}$ \\
\hline total trigger rate & $\sim 6 \cdot 10^{4}$ events/s (trigger in) \\
& $\sim 3 \cdot 10^{4}$ events/s (trigger out) \\
\hline coincidence rate per bin & $0.05-0.1$ breakup events/s \\
\hline$\Delta \sigma / \sigma$ of breakup events & $\sim 1 \%$ \\
\hline collected data & $22 \mathrm{~TB}$ \\
\hline
\end{tabular}

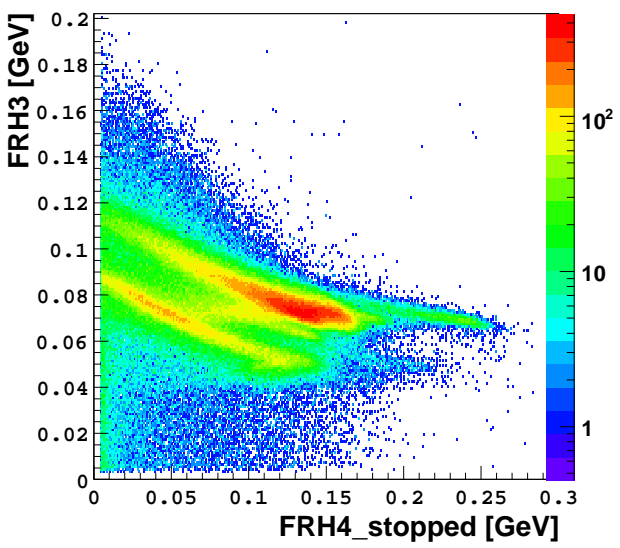

Figure 2. Example of the $\Delta E-E$ identification spectrum obtained with the use of the Forward Detector. Almost all deuterons from elastic dp scattering are stopped in the fourth plane of The Forward Range Hodoscope (FRH).

After selection of the proton-proton coincidences and having performed the energy calibration, the differential cross section of the breakup reaction can be determined for a chosen kinematic configuration. The configuration is defined by the emission angles of the two outgoing protons: two polar angles $\theta_{1}$ and $\theta_{2}$ and the relative azimuthal angle $\phi_{12}$ (reconstructed from FPC in Forward Detector and MDC in Central Detector). The kinematical spectra $E_{1} v s E_{2}$ are calculated for each analyzed configuration with the defined integratation limits of the two polar angles and relative azimuthal angle (Figure 3).

For a chosen configuration events are projected onto the kinematical curve corresponding to the point-like, central geometry. The rate of events is obtained as function of the arc-length $S$ measured along the kinematic curve (Figure 4). These rates have to be normalized using rates of the elastic scattering events and the known cross-section for this process [7].

The data analysis will be continued with the aim to detemine the differential cross sections for the the deuteron breakup process in $d+p$ system at energies of 340,380 and $400 \mathrm{MeV}$. The data will be compared to the theoretical predictions for three nucleon $(3 N)$ systems with the aim to investigate relativistic effects. 

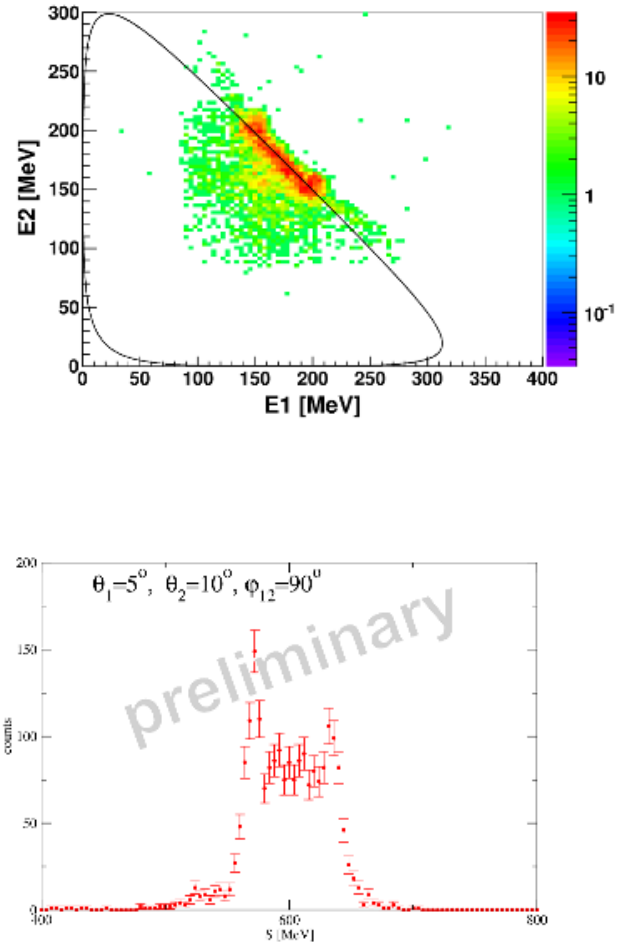

Figure 3. $E_{1}-E_{2}$ coincidence spectrum of the two protons detected at $\theta_{1}=5^{\circ} \pm 1^{\circ}, \theta_{2}=10^{\circ} \pm 1^{\circ}$, and $\phi_{12}=90^{\circ} \pm 5^{\circ}$. The solid line shows a three-body kinematical curve, calculated for the central values of the experimental angular ranges.
Figure 4. An example of the non-normalized rate of breakup events obtained for the chosen kinematical configuration $\left(\theta_{1}=5^{\circ} \pm 1^{\circ}, \theta_{2}=10^{\circ} \pm 1^{\circ}\right.$, and $\left.\phi_{12}=90^{\circ} \pm 5^{\circ}\right)$ as a function of the $S$ value (arc-lenght along the kinematics with the starting point at $E_{2}$ minimum).

\section{Acknowledgments}

This work has been supported by FFE funds of the Forschunszentrum Jülich; by the European Commision under the 7th Framework Programme through the "Research Infrastructures" action of the Capacities Programme (Call: FP7-INFRASTRUCTURES-2008-1), the Foundation for Polish Science MPD program, co-financed by the European Union within the European Regional Development Fund, Małopolskie Centrum Przedsiębiorczości - Project "Doctus - Małopolski fundusz stypendialny dla doktorantów", the Polish 2013- 2015 science founds as research Project No. 2012/05/E/ST2/02313 and funding from Jagiellonian University within SET project - the project is co-financed by the European Union.

\section{References}

[1] W. Glöckle et al., Phys. Rep. 274, 107 (1996).

[2] A. Deltuva, K. Chmielewski and P.U. Sauer, Phys. Rev. C67, 034001 (2003);

A. Deltuva, R. Machleidt and P.U. Sauer, Phys. Rev. C68, 024005 (2003).

[3] E. Epelbaum, Rep. Prog. Nucl. Phys. 57, 654 (2006).

[4] S. Kistryn and E. Stephan, J. Phys. G: Nucl. Part. Phys. 40063101 (2013).

[5] H. Witała, R. Skibinski and J. Golak, The European Physical Journal, A30,369 (2006).

[6] H. Witała et al. Phys. Rev. C83, 044001 (2011).

[7] K. Ermisch et al. Phys. Rev. C68, 051001(R) (2003). 